\title{
Barriers to the Treatment of Hepatitis C
}

\section{Patient, Provider, and System Factors}

James A. Morrill, MD, PhD, ${ }^{1,2}$ Melissa Shrestha, BS, CHES, ${ }^{3}$ Richard W. Grant, MD, MPH

${ }^{1} G e n e r a l$ Medicine Division, Department of Medicine, Massachusetts General Hospital, Boston, MA, USA; ${ }^{2} \mathrm{MGH}$ Charlestown

HealthCare Center, Charlestown, MA, USA; ${ }^{3} \mathrm{MGH}$ Revere HealthCare Center, Revere, MA, USA.

BACKGROUND: Hepatitis C virus (HCV) infection is both prevalent and undertreated.

OBJECTIVE: To identify barriers to HCV treatment in primary care practice.

DESIGN: Cross-sectional study.

SETTING AND PARTICIPANTS: A cohort of $208 \mathrm{HCV}$-infected patients under the care of a primary care physician (PCP) between December 2001 and April 2004 at a single academically affiliated community health center.

MEASUREMENTS: Data were collected from the electronic medical record (EMR), the hospital clinical data repository, and interviews with PCPs.

MAIN RESULTS: Our cohort consisted of 208 viremic patients with $\mathrm{HCV}$ infection. The mean age was $47.6( \pm 9.7)$ years, $56 \%$ were male, and $79 \%$ were white. Fifty-seven patients $(27.4 \%$ of the cohort) had undergone HCV treatment. Independent predictors of not being treated included: unmarried status (adjusted odds ratio [aOR] for treatment 0.36, $P=.02$ ), female gender (aOR 0.31, $P=.01$ ), current alcohol abuse (aOR 0.08, $P=.0008$ ), and a higher ratio of no-shows to total visits (aOR 0.005 per change of 1.0 in the ratio of no-shows to total visits, $P=.002$ ). The major PCP-identified reasons not to treat included: substance abuse $(22.5 \%)$, patient preference $(16 \%)$, psychiatric comorbidity $(15 \%)$, and a delay in specialist input (12\%). For $13 \%$ of the untreated patients, no reason was identified.

CONCLUSIONS: HCV treatment was infrequent in our cohort of outpatients. Barriers to treatment included patient factors (patient preference, alcohol use, missed appointments), provider factors (reluctance to treat past substance abusers), and system factors (referral-associated delays). Multimodal interventions may be required to increase $\mathrm{HCV}$ treatment rates.

KEY WORDS: hepatitis $\mathrm{C}$ virus infection; interferon and ribavirin therapy; barriers to treatment; substance abuse.

DOI: $10.1111 / \mathrm{j} .1525-1497.2005 .0161 . x$

J GEN INTERN MED 2005; 20:754-758.

$\mathrm{H}$ epatitis $\mathrm{C}$ virus (HCV) infection is the most prevalent blood-borne disease in the United States, affecting approximately $2 \%$ of the U.S. population. ${ }^{1}$ Despite current limitations of available therapy, ${ }^{2-5}$ antiviral treatment represents the only means of preventing serious HCV-associated liver disease. Studies in various patient populations-including patients referred to an urban hepatology clinic, primary care patients in Rochester, Minn, and HCV-positive veterans-have suggested that $73 \%$ to $87 \%$ of patients presenting with confirmed $\mathrm{HCV}$ infection go untreated, markedly reducing the true

The authors have no conflicts of interest to declare for this article or this research.

This work was presented previously in abstract form at the 2004 national meeting of Society for General Internal Medicine, Chicago, IL.

Address correspondence and requests for reprints to Dr. Morrill: Adult Medicine Unit, MGH Charlestown HealthCare Center, 73 High Street, Charlestown, MA 02129 (e-mail: jmorrill@partners.org). impact of interferon (IFN)-based therapy (which has 50\% success rate in clinical trial populations and a far lower success rate in other groups). ${ }^{6-8}$ Explanations offered for these low treatment rates have included patient preference, medical and psychiatric comorbidity, and recent substance abuse. However, recent feasibility studies in active injection drug users, mentally ill patients, prisoners, and participants in methadone maintenance programs have demonstrated that treatment can be successful in patients once considered too difficult to treat. ${ }^{9-12}$ Based on these findings, the most recent National Institutes of Health (NIH) guidelines for the management of HCV infection now advocate for an inclusive approach to treatment, urging that efforts be made to increase the availability of the best current treatment for patients who have traditionally been ineligible for clinical trials. ${ }^{13}$ Thus, identifying and working to overcome barriers to HCV treatment have become important goals in approaching the HCV epidemic.

We undertook a cross-sectional study of all patients cared for at an urban community health center with a positive HCV serology and a positive HCV viral load. The goals of our study were to: (a) describe the characteristics of our HCV-infected population; (b) define the proportion of this population that has undergone antiviral treatment; and (c) identify in detail the barriers to treatment. We hypothesized that barriers to treatment would include patient factors (such as patient preference, comorbidities, and follow-up), provider factors (such as providers' perceptions of contraindications to treatment), and system factors (such as type of insurance coverage and communication with specialists). The overarching goal of our study was to identify potentially modifiable barriers as targets for future interventions to increase the applicability, and thus the net effectiveness, of antiviral treatment.

\section{METHODS}

\section{Setting}

This study was undertaken at the Massachusetts General Hospital (MGH) Revere HealthCare Center, an urban community health center in Revere, MA, affiliated with MGH. Revere (population 47,283) is located in a Health Professionals Shortage Area for primary care 5 miles north of Boston, Mass. More than $26 \%$ of Revere residents live below $200 \%$ of the federal poverty level. According to 2000 census data, $83 \%$ of the residents were white, 9\% Latino, 5\% Asian, and 3\% African American. Physicians at the health center rely exclusively on an electronic medical record (EMR) for patient care.

Received for publication March 18, 2005

and in revised form March 21, 2005

Accepted for publication March 21, 2005 


\section{Patients}

We retrospectively created a HCV registry for all patients with at least 1 encounter (physician or nurse visit, laboratory test, or radiology examination) at the health center between December of 2001 and April of 2004 who had a positive anti-HCV antibody test $(N=412)$. From this initial list, we used EMR review and individual meetings with each of the health center's primary care providers to identify the subset of patients under the care of a primary care provider at the health center at some point during the study period (with at least 1 primary care visit during that time; $N=259$ ). After excluding patients with negative HCV viral load, our analytic cohort consisted of 208 primary care patients with active HCV infection.

\section{Data Collection}

We performed electronic queries to define demographic characteristics and determine comorbid conditions, clinic visit type and frequency, and laboratory values relevant to HCV infection (HCV viral load, alanine aminotransferase [ALT] level, and HCV genotype). For each patient, we collected the last measured laboratory results at the time of treatment status determination. Detailed information about medical and psychiatric history, social and substance abuse history, treatment status, and contact with providers at the health center was obtained from manual structured chart abstraction and review of all written notes in the EMR for the time period between December 2001 and April 2004. Medical comorbidity was quantified using a version of the Charlson Comorbidity Index, in which 1 to 6 points are assigned for each of 17 diagnosis categories covering most major chronic diseases (e.g., coronary artery disease, diabetes, renal failure, chronic liver disease, malignancy, AIDS, and others). ${ }^{14}$ For the 151 patients who had not received treatment for their HCV infection, the providers' opin- ions of why they had not been treated were deduced from clinic notes. For 98 of these 151 patients, we confirmed or revised our determination of the provider's opinion based on a 1-on-1 interview with the provider. This study was approved by the MGH Institutional Review Board.

\section{Statistical Methods}

In this cross-sectional analysis, treated patients were compared with untreated patients. Continuous variables were compared using $t$ tests if normally distributed and the Wilcoxon rank sum if non-normal. Categorical variables were compared using $\chi^{2}$ tests. Demographic and clinical variables that were significant based on univariate analyses were subsequently entered into stepwise logistic regression models with backward elimination to determine adjusted odds ratios (aORs) of HCV treatment. Gender was also included in the multivariate analysis because of its importance as a variable. All analyses were performed using SAS (SAS version 8.0, SAS Institute, Cary, NC). $P$ values of less than .05 were considered statistically significant.

\section{RESULTS}

\section{Characteristics of the Study Cohort}

We identified 259 patients with a positive HCV serology under the care of a primary care physician (PCP) at the MGH Revere HealthCare Center between December 2001 and April of 2004. Of these, 208 patients also had a positive HCV viral load and were studied further. As shown in Table 1, the viremic patients had a mean age of 47.6 years (standard deviation [SD] \pm 9.7 ). The majority (78.9\%) were white, $63.5 \%$ were unmarried, and $55.8 \%$ were male; the most significant minority group in the cohort were Asian Americans (10.1\%), most of whom were part

Table 1. Demographics, CoMorbid Conditions, and Laboratory Values of the Cohort of HCV-Positive, Viremic Patients

\begin{tabular}{|c|c|c|c|c|}
\hline & Treated $(n=57)$ & Untreated $(n=151)$ & Total Cohort $(n=208)$ & $P$ Value* \\
\hline Age, mean (SD) & $47.4(7.0)$ & $47.7(10.5)$ & $47.6(9.7)$ & .79 \\
\hline Male, $n(\%)$ & $36(63.2)$ & $80(53.0)$ & $116(55.8)$ & .22 \\
\hline \multicolumn{5}{|l|}{ Race, $n(\%)$} \\
\hline White & $40(70.2)$ & $124(82.1)$ & 164 (78.9) & .06 \\
\hline Asian & $7(12.3)$ & $14(9.3)$ & $21(10.1)$ & .52 \\
\hline Other & & & $23(11.1)$ & \\
\hline Married, $n(\%)$ & $31(54.4)$ & $45(29.8)$ & $76(36.5)$ & .001 \\
\hline Employed, $n(\%)$ & $26(45.6)$ & $84(41.1)$ & $88(42.3)$ & .55 \\
\hline Private insurance, $n(\%)$ & 25 (43.9) & $58(38.4)$ & 83 (39.9) & .47 \\
\hline Methadone maintenance, $n(\%)$ & $7(12.3)$ & $25(16.6)$ & $32(15.4)$ & .44 \\
\hline History of incarceration, $n(\%)$ & $1(1.8)$ & $11(7.3)$ & $12(5.8)$ & .13 \\
\hline Concurrent HBV or HIV infection, $n(\%)$ & $1(1.8)$ & $12(8.0)$ & $13(6.3)$ & .10 \\
\hline Major psychiatric disorder, $n(\%)$ & $24(42.1)$ & $91(60.3)$ & $115(55.3)$ & .02 \\
\hline Referred to GI specialist, $n(\%)$ & $49(86.0)$ & $79(52.3)$ & $128(61.5)$ & $<.0001$ \\
\hline Charlson comorbidity score, mean (SD) & $1.16(1.36)$ & $1.52(1.80)$ & $1.42(1.69)$ & .12 \\
\hline $\mathrm{HCV}$ viral load (IU/mL; $N=153$ ) Mean (SD) & $144,359(232,404)$ & $426,646(535,266)$ & $329,224(429,968)$ & $<.0001$ \\
\hline ALT (IU/L), Mean (SD) & $52.0(69.1)$ & $72.7(68.5)$ & $67.1(68.7)$ & .05 \\
\hline \multicolumn{5}{|l|}{ HCV genotype $(N=67)$} \\
\hline 1 & $20(64.5)$ & $20(55.6)$ & $40(59.7)$ & .46 \\
\hline 2,3 , or 4 & $11(35.5)$ & $16(44.4)$ & $27(40.3)$ & \\
\hline
\end{tabular}

Numbers are means and standard deviations (SD), or number and proportions. Private insurance includes any insurance plan other than Medicaid (MassHealth), Medicare, Massachusetts Free Care, or Self-pay. Major psychiatric disorder includes depression, bipolar disease, anxiety, schizophrenia, or posttraumatic stress disorder. The Charlson comorbidity score is a weighted composite of 17 major medical conditions (see Methods section for details). IU, international units; L, liter; $m L$, milliliter; HCV, hepatitis $C$ virus; HBV, hepatitis B virus; HIV, human immunodeficiency virus; ALT, alanine aminotransferase; GI, General Internist.

${ }^{*} \mathrm{P}$ values are for univariate comparisons between treated and untreated patients $\chi^{2}$ test for categorical variables; Student $\mathrm{t}$ test for continuous variables). 
of the Cambodian refugee community in Revere. Many members of the cohort were disadvantaged: $57.7 \%$ were unemployed, $60.1 \%$ lacked private health insurance, $15.4 \%$ had a history of methadone treatment for opioid addiction, and $5.8 \%$ had a history of incarceration. We found a relatively low level of medical comorbidity (mean Charlson comorbidity score $=1.42$ ), although $6.3 \%$ of participants were coinfected with either hepatitis B virus (HBV) or human immunodeficiency virus (HIV). Major psychiatric disease (defined as treated depression, bipolar disorder, anxiety, schizophrenia, or posttraumatic stress disorder) was present in over half of the cohort (55.3\%). The mean HCV viral load was $329,224 \mathrm{IU} / \mathrm{mL}$ ( $\mathrm{SD} \pm 429,968)$, and the mean ALT value was $67.1 \mathrm{U} / \mathrm{L}$ (SD \pm 68.7 ). For the 67 patients $(32.2 \%$ of cohort) with $\mathrm{HCV}$ genotype results, $59.7 \%$ were infected with $\mathrm{HCV}$ genotype 1.

\section{Univariate Analysis of Treated Versus Untreated Patients}

Of the 208 subjects, $128(61.5 \%)$ were referred to a treating specialist, but only 57 (27.4\%) had undergone HCV treatment. Table 1 compares the characteristics of treated and untreated patients. Treated patients were significantly more likely than untreated patients to be married (54.4\% vs $29.8 \%, P=.001)$ and less likely to have a major psychiatric disorder $(42.1 \%$ vs $60.3 \%, P=.02$ ). There were no significant differences in age, gender, racial composition, employment status, type of insurance, HIV or HBV status, or history of incarceration or methadone maintenance between treated and untreated patients. Compared with treated patients, untreated patients had a significantly higher mean HCV viral load (426,646 vs 144,359 IU/ $\mathrm{mL}, P<.0001)$ and a significantly higher mean ALT value $(72.7$ vs $52.0 \mathrm{IU} / \mathrm{L}, P=.05)$, although the number in each group with $\mathrm{HCV}$ genotype 1 infection did not differ.

Current and past substance abuse was significantly more frequent among untreated patients than among treated patients (Fig. 1). Nearly one quarter (23.8\%) of untreated patients were currently abusing alcohol, compared with $5.3 \%$ of treated patients $(P=.002)$. There was a similar $>4$-fold difference between treated and untreated patients in the proportion of patients currently abusing drugs other than alcohol $(P=.03)$. Past alcohol or drug abuse was common among both treated and untreated patients, although only past non-alcohol drug

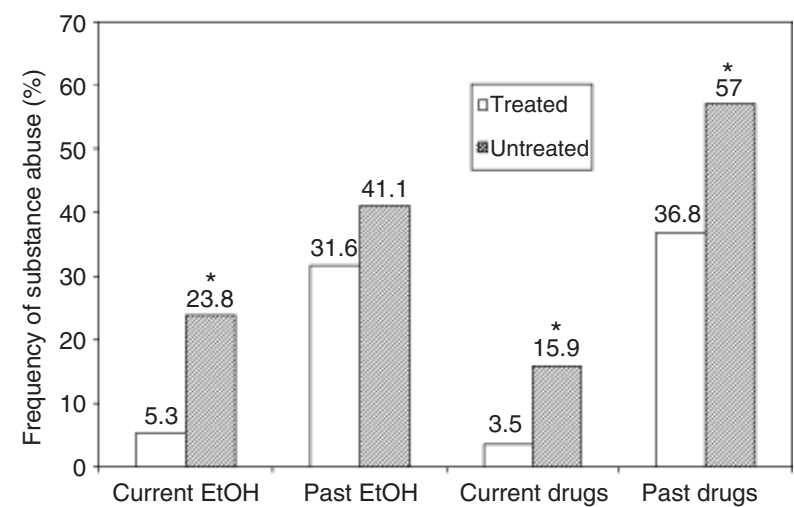

FIGURE 1. Frequency of substance abuse among treated (white bars) and untreated patients (shaded bars). EłOH, alcohol abuse; drugs, current abuse of any drug other than alcohol. ${ }^{*} P<.05 ; \chi^{2}$ test between treated and untreated patients.
Table 2. Multivariate Analysis of Patient Factors Affecting the Probability of Receiving HCV Treatment

\begin{tabular}{lccc}
\hline \hline Patient characteristic & $\begin{array}{c}\text { Adjusted Odds Ratio } \\
\text { for Receiving } \\
\text { Treatment }\end{array}$ & $\begin{array}{c}95 \% \\
\text { Confidence } \\
\text { Interval }\end{array}$ & $\begin{array}{c}\boldsymbol{P} \\
\text { Value }\end{array}$ \\
\hline Married status & 2.79 & 1.15 to 6.76 & .02 \\
Female gender & 0.31 & 0.12 to 0.79 & .01 \\
Current EtOH abuse & 0.08 & 0.02 to 0.35 & .0008 \\
Total no-shows/total & $0.005^{*}$ & $<0.001$ to 0.14 .002 \\
$\quad$ visits & & & \\
\hline
\end{tabular}

EtOH, alcohol.

* Odds ratio is per increase of 1.0 in the total no-show/total visit ratio.

abuse was significantly more prevalent in untreated than in treated patients $(P=.01)$.

Untreated patients were also significantly more likely to miss clinic visits than treated patients; the proportion of no shows out of total clinic visits was $25 \%( \pm 17 \%)$ for untreated patients, versus $16 \%$ ( $\pm 14 \%$ ) for treated patients $(P<.0005)$. However, untreated patients were not significantly more likely to attend the clinic on a "walk-in" basis than treated patients; the proportion of MD visits that were unscheduled walk-in visits was $14 \%( \pm 19 \%)$ for untreated patients versus $10 \%$ $( \pm 12 \%)$ for treated patients $(P=.07)$.

\section{Multivariate Analysis}

After controlling for 2 measures of disease severity (viral load and hepatic enzyme abnormalities), the following variables remained independently associated with treatment status in logistic regression models: married status (aOR for treatment $2.79,95 \%$ confidence interval [CI] 1.15 to $6.76, P=.02$ ), female gender (aOR $0.31,95 \% \mathrm{CI} 0.12$ to $0.79, P=.01$ ), current alcohol abuse (aOR 0.08, 95\% CI 0.02 to $0.35, P=.0008$ ), and the ratio of no-shows to total clinic visits (aOR per increase of 1.0 in the ratio $0.005,95 \% \mathrm{CI}<0.001$ to $0.14, P=.002$ ) (Table 2). Current and past non-alcohol drug abuse and the presence of a major psychiatric disorder were not independently associated with treatment status.

\section{PCP-Identified Reasons for Untreated Status}

For the 151 untreated patients, we reviewed all clinic notes over the preceding 2 years to identify PCP-documented reasons for why the patients had not been treated (Table 3). For a

Table 3. PCP-Identified Reasons why Patients were not Treated

\begin{tabular}{lr}
\hline \hline Characteristic & $n(\%)$ \\
\hline Substance abuse & $34(22.5)$ \\
Patient preference & $24(16.1)$ \\
Psychiatric factors & $23(15.2)$ \\
No clearly identified reason & $20(13.2)$ \\
Delays in specialist input & $18(11.9)$ \\
Lost to follow-up & $12(7.9)$ \\
Medical factors & $12(7.9)$ \\
Other factors & $8(5.3)$ \\
Total & $151(100)$ \\
\hline
\end{tabular}

PCP, primary care physician. Substance abuse is defined by each PCP and includes past abuse (15 patients, 9.9\%) and present abuse (19 patients, 12.6\%). Psychiatric and medical factors are as defined by each $P C P$. "Other factors" include (a) social instability and (b) normal liver function tests despite a positive viral load. 
majority of these patients (98 of 151), clinic note review was augmented by 1-on-1 interviews with the patients' PCPs. The most common reasons provided by PCPs for why patients were not treated included: past and present substance abuse (22.5\% of patients-including $9.9 \%$ with past and $12.6 \%$ with current substance abuse); patient preference against being treated (16\%); psychiatric and medical comorbidity $(15.2 \%$ and $7.9 \%$, respectively); delays in obtaining input from a specialist (11.9\%); and loss to follow-up (7.9\%). For 20 patients (13.2\%), no reason not to treat was identified by the PCP.

\section{DISCUSSION}

In our cross-sectional, clinic-based analysis of HCV infection, we found that only $27 \%$ of viremic patients receiving primary care at an academically affiliated community health center had undergone treatment. The cohort as a whole was predominantly middle-aged, white, unmarried, and disadvantaged (e.g., often unemployed and not covered by private health insurance). Significant predictors of not being treated included unmarried status and female gender as well as current alcohol abuse and suboptimal clinic attendance behavior. Among the untreated patients, PCPs identified past or present substance abuse, patient preference, psychiatric and medical comorbidity, and delays in obtaining specialist input as the principal reasons why their patients did not receive antiviral therapy.

Of the 259 patients initially identified as having an antiHCV antibody by Enzyme Immunoassay (EIA) test, only 208 patients (80\% of the total) had a positive viral load and were thus included in the study. This $20 \%$ "false-positive rate" is high compared with the $3 \%$ to $10 \%$ seen in studies of patients referred to specialized hepatitis C clinics, ${ }^{6,7}$ and is higher than the rate of $14 \%$ reported in a primary care-based study of $\mathrm{HCV}$ patients in Rochester, Minn (in which a recombinant immunoblot assay (RIBA) was used instead of the HCV viral load as the confirmatory test), ${ }^{8}$ although it is lower than the rate of $26 \%$ reported in the NHANES III, a survey of unselected U.S. households. ${ }^{1}$ There are several possible sources of "false-positive" antibody tests, including true clearance of the HCV virus (which can occur in up to $15 \%$ to $25 \%$ of patients who initially contract $\mathrm{HCV}$ infection ${ }^{2}$ ) and a false-positive EIA Test. Because few of our patients underwent confirmatory antibody testing with the more specific RIBA antibody assay, we could not distinguish between these possibilities. However, because of the relatively low pretest probability of HCV infection in our primary care population, the number of false-positive EIAs in our sample may indeed have been high. Indeed, the 208 patients with confirmed HCV infection represent only $1.3 \%$ of our total adult clinic population of approximately 16,000-a proportion consistent with the prevalence of viremic HCV infection in the general U.S. population estimated in the NHANES study. ${ }^{1}$ Thus, our findings provide further support for the 2004 U.S. Preventive Services Table Force (USPSTF) guideline recommendation against routine screening for $\mathrm{HCV}$ infection in asymptomatic adults in the general population. ${ }^{15,16}$

The clinical predictors and PCP-identified barriers to $\mathrm{HCV}$ treatment identified in our study suggest that obstacles to treatment exist at the level of patients, providers, and the system as a whole. Patient barriers included their own preference against treatment, ongoing alcohol abuse, and poor follow-up behavior. Although providers cited medical and psychiatric co- morbidity as barriers to treatment, these variables did not significantly correlate with treatment status in our analysis. A tendency not to treat patients with a past history of substance abuse may have represented a provider-level barrier; there was no evidence of treatment disparities based on age, gender, ethnicity, employment status, use of methadone maintenance, or a history of incarceration. On the system level, providers cited delays in communication with specialists as a significant reason why patients were not treated, whereas type of insurance (private vs "safety net"-i.e., Medicaid, Medicare, Massachusetts Free Care, or Self-Pay) did not appear to influence treatment status.

Some experts have argued that the percentage of treated patients should be low given the arduous nature and cost of current treatment protocols. ${ }^{17}$ Moreover, there are a number of medical and psychiatric conditions that are absolute or relative contraindications to IFN/ribavirin treatment, including significant anemia, current alcohol use, active coronary artery disease, active autoimmune disease, pregnancy, diabetes, and suicidal or psychotic depression. ${ }^{2,4,13,18}$ We estimate that slightly over one-third of our untreated cohort had appropriate "barriers" to treatment, defined as current substance abuse $(12.6 \%)$, psychiatric comorbidity (15.2\%), or medical comorbidity $(7.9 \%)$. Interestingly, there was no difference between treated and untreated patients in the frequency of $\mathrm{HCV}$ genotype 1 relative to the other genotypes, despite the fact that genotype 1 infection requires a longer duration of treatment and has lower virologic response rates than the other genotypes $^{3,5,13}$ - data that have made genotype 1 infection a significant barrier to completing treatment in other populations. ${ }^{6}$

For the remaining two thirds of untreated patients, barriers to treatment may be at least partially surmountable. As seen in a recent survey of injection drug users in Rhode Island, ${ }^{19}$ patient reluctance is a difficult barrier to overcome but may be amenable to systematic patient outreach, education about treatment, and a supportive treatment program. Similarly, poor follow-up may respond to frequent visits, collaboration with nurses or case managers, or a system of directly observed therapy, as has been seen with tuberculosis and HIV treatment. ${ }^{20,21}$ The common bias against treating past (nonalcohol) substance abusers may reflect prior national guidelines, which recommended excluding such patients from treatment until they had been abstinent for at least 6 months ${ }^{22}$; while the appropriateness of treating such patients has continued to be debated, ${ }^{17,23}$ the feasibility of treating past and even current opioid abusers has been suggested in small trials, ${ }^{10,11}$ new data that have been acknowledged in the most recent NIH guidelines. ${ }^{13}$ The delays associated with specialist referral qualitatively included delays in getting to the specialist's office (determined both by waiting times and by patient no-shows) and the time taken by the specialist to decide whether a patient was appropriate for treatment; such delays were likely enhanced by the fact that at our institution, the locus of HCV treatment is generally at a specialized hepatology clinic located at the main hospital, a significant distance away from the health center. Such delays may be overcome by establishing closer collaborations between hepatologists and primary care doctors, or, when feasible, moving HCV treatment into the primary care setting, as has been piloted. ${ }^{24}$ Finally, our data suggest that $13.2 \%$ of the untreated patients (20 patients) had no identifiable barriers to treatment. Clinic-based registries of all $\mathrm{HCV}$-positive patients may represent one 
means of identifying such patients for further assessment and possible treatment.

Our study has several limitations. First, our analysis represents the experience for a single academically affiliated community health center serving a predominantly low socioeconomic white population and may therefore be less generalizable to other patient settings. For example, our cohort contains few AfricanAmerican patients, and therefore our analysis does not capture the special barriers faced by this population, which has a lower rate of response to antiviral therapy and has been poorly represented in the major registration trials of HCV therapy. ${ }^{25}$ Second, we collected only limited information on the socioeconomic status of the study patients. Unmeasured socioeconomic variables such as income or education level may also have predicted treatment status and may be correlated with other barriers reported in our study, such as clinic no-show behavior and loss to follow-up. Third, while our cross-sectional design provides important information on the association between demographic and clinical factors and HCV treatment status, we were unable to assess trends for individual patients over time. Fourth, our electronic data queries did not include data on the frequency and outcome of liver biopsies, which are sometimes an important hidden barrier to proceeding with treatment. Finally, our provider interviews were limited in scope; while we used them to refine our database of $\mathrm{HCV}$-positive patients and in some cases to gain further information on why patients had not been treated, we did not assess the attitudes, knowledge, or beliefs of the providers.

Although the current rate of HCV treatment remains low, a number of the obstacles to HCV treatment may be amenable to change. These modifiable barriers include negative views of patients about treatment, inadequate patient follow-up with primary care providers, a tendency for providers not to consider treatment of past substance abusers, and delays in obtaining the input of specialists. Efforts to overcome these barriers require patient and provider education, interventions to improve clinic attendance, and collaboration between PCPs and both mental health and substance abuse professionals. Reducing the distance between PCPs and those directly involved in HCV treatment by introducing $\mathrm{HCV}$ treatment into the primary care clinic represents another system-level strategy. A comprehensive HCV disease management model addressing each of these barriers, analogous to those that have improved disease outcomes for other prevalent conditions such as diabetes, ${ }^{26}$ may offer the most effective solution to low HCV treatment rates and may thus raise the overall efficacy of treatment.

This work was supported by the Massachusetts General Hospital Clinical Research Program and by MGH Community Health Associates.

\section{REFERENCES}

1. Alter M, et al. The prevalence of hepatitis $C$ virus infection in the United States, 1988 through 1994. N Engl J Med. 1999;341:556-62.

2. Flamm S. Chronic hepatitis c virus infection. JAMA. 2003;289: 2413.

3. Fried MW, et al. Peginterferon alfa-2a plus ribavirin for chronic hepatitis C virus infection. N Engl J Med. 2002;347:975-82.

4. Lauer G, Walker B. Medical progress: hepatitis c virus infection. N Engl J Med. 2001;345:41.

5. Manns MP, et al. Peginterferon-alpha2b plus ribavirin compared with interferon-alpha2b plus ribavirin for initial treatment of chronic hepatitis C: a randomised trial. Lancet. 2001;348:958-65.

6. Cawthorne CH, et al. Limited success of HCV antiviral therapy in United States veterans. Am J Gastroenterol. 2002;97:149-55.

7. Falck-Ytter $\mathbf{Y}$, et al. Surprisingly small effect of antiviral treatment in patients with hepatitis C. Ann Intern Med. 2002;136:288-92.

8. Yawn BP, et al. Identification and management of hepatitis $\mathrm{C}$ patients in primary care clinics. J Fam Pract. 2002;51:135-40.

9. Allen SA, et al. Treatment of chronic hepatitis C in a state correctional facility. Ann Intern Med. 2003;138:187-90.

10. Backmund $\mathbf{M}$, et al. Treatment of hepatitis $\mathrm{C}$ infection in injection drug users. Hepatology. 2001;34:188-93.

11. Sylvestre D. Treating hepatitis $\mathrm{C}$ in methadone maintenance patients: an interim analysis. Drug Alcohol Dependence. 2002;67:117-23.

12. Thiel DHV, et al. Interferon-alpha can be used successfully in patients with hepatitis $\mathrm{C}$ virus-positive chronic hepatitis who have a psychiatric illness. Eur J Gastroenterol Hepatol. 1995; 7:165-8.

13. Anonymous. Management of hepatitis c: 2002. NIH Consens State Sci Statements. 2002;19:1-46.

14. Librero J, Peiro S, Ordinana R. Chronic comorbidity and outcomes of hospital care: length of stay, mortality, and readmission at 30 and 365 days. J Clin Epidemiol. 1999;52:171-9.

15. Chou R, Clark EC, Helfand M. Screening for hepatitis C virus infection: a review of the evidence for the US preventive services task force. Ann Intern Med. 2004; 140:465-79.

16. USPSTF. Screening for hepatitis $\mathrm{C}$ virus infection in adults: recommendation statement. Ann Intern Med. 2004;140:462-4.

17. Davis GL, Rodrigue JR. Treatment of chronic hepatitis $\mathrm{C}$ in active drug users. N Engl J Med. 2001;345:215-7.

18. Schiff E. The alcoholic patient with hepatitis C virus infection. Am J Med. 1999; 107:95S-9S.

19. Stein MD, Maksad J, Clarke J. Hepatitis C disease among injection drug users: knowledge, perceived risk and willingness to receive treatment. Drug Alcohol Dependence. 2001;61:211-5.

20. Bamberger JD, et al. Helping the urban poor stay with antiretroviral HIV drug therapy. Am J Public Health. 2000;90:699-701.

21. Smirnoff $\mathbf{M}$, et al. Directly observed therapy in an inner city hospital. Int J Tuberc Lung Dis. 1998;2:134-9.

22. Anonymous. Management of hepatitis C. NIH Consens Statements. 1997; 15:1-41.

23. Edlin BR, et al. Is it justifiable to withhold treatment for hepatitis $\mathrm{C}$ from illicit-drug users? N Engl J Med. 2001;345:211-4.

24. McGinn T. How primary care enhances hepatitis C treatment (conference workshop). J Gen Intern Med. 2004;19:11.

25. Strader DB. Understudied populations with hepatitis C. Hepatology. 2002;36:S226-36.

26. Grant RW, et al. Impact of population management with direct physician feedback on care of patients with type 2 diabetes. Diabetes Care. 2003; 26:2275-80. 